\title{
SELETIVIDADE DE PRODUTOS FITOSSANITÁRIOS UTILIZADOS NA CULTURA DO PESSEGUEIRO A Trichogramma pretiosum RILEY, 1879 (Hymenoptera: Trichogrammatidae)
}

\author{
Selectivity of pesticides used in peach orchard to Trichogramma pretiosum \\ Riley, 1879 (Hymenoptera: Trichogrammatidae)
}

\author{
Fabrizio Pinheiro Giolo', Anderson Dionei Grützmacher², Cristiane Gindri Manzoni', \\ Crislaine Alves Barcellos de Lima ${ }^{3}$, Wagner da Roza Härter ${ }^{4}$, Cristiane Müller ${ }^{5}$
}

\begin{abstract}
RESUMO
A seletividade de dez formulações comerciais de produtos fitossanitários utilizados na cultura do pessegueiro foi avaliada em laboratório $\left(25 \pm 1{ }^{\circ} \mathrm{C}\right.$, UR $70 \pm 10 \%$ e fotofase de 14 horas), através da exposição de adultos de Trichogramma pretiosum Riley a resíduos secos dos produtos pulverizados sobre placas de vidro. O número de ovos parasitados por fêmea, foi mensurado e a redução no parasitismo (RP), em relação à testemunha (água), foi utilizada para classificar os compostos em: inócuos (<30\% RP), levemente nocivos (30-79\% RP), moderadamente nocivos (80-99\% RP) e nocivos (>99\% RP). Os produtos fitossanitários captana, ditianona, mancozebe, metoxifenozida e óxido cuproso foram inócuos; glifosato e óleo mineral foram levemente nocivos e abamectina, cianamida e etofenproxi foram nocivos a $T$. pretiosum
\end{abstract}

Termos para indexação: Agrotóxicos, parasitóide de ovos, controle biológico, Prunus persica L.

\begin{abstract}
The selectivity of ten commercial pesticide formulations used in peach orchard was evaluated in laboratory tests $\left(25 \pm 1{ }^{\circ} \mathrm{C}\right.$, RH $70 \pm 10 \%$ and 14 hours photophase) by exposing the adult of Trichogramma pretiosum Riley to fresh dry pesticide film applied on glass plates. The number of eggs parasitized per adult female and the reduction in parasitism (RP) compared to control (treated with water) were used to measure the effect of the chemical in harmless ( $<30 \% \mathrm{RP})$, slightly harmful (30-79\% RP), moderately harmful (80-99\% RP) and harmful (>99\% RP). The pesticides captan, copper oxide, dithianon, mancozeb and methoxyfenozide were harmless; gliphosate and mineral oil was slightly harmful and abamectin, cyanamide and etofenprox were harmful to T. pretiosum.
\end{abstract}

Index terms: Pesticides, egg parasitoid, biological control, Prunus persica $\mathrm{L}$.

\section{(Recebido em 22 de novembro de 2005 e aprovado em 24 de agosto de 2006)}

\section{INTRODUÇÃO}

O Estado do Rio Grande do Sul é o principal produtor de pêssegos do Brasil, sendo responsável por $50 \%$ da produção nacional. Por outro lado, as regiões produtoras de pêssego, no Sul do Brasil, são caracterizadas pelo baixo rendimento da cultura em relação a outros Estados brasileiros (IBGE, 2005), principalmente devido às condições climáticas favoráveis ao estabelecimento de pragas, promovendo a intensificação na utilização de produtos fitossanitários pelos persicultores.

Em relação aos insetos-praga dessa cultura, a grafolita ou broca-dos-ponteiros, Grapholita molesta (Busck, 1916) (Lepidoptera: Tortricidae), destaca-se como uma das principais, ocasionando perdas significativas à cultura do pessegueiro, no Rio Grande do Sul (BOTTON et al., 2001). Mais recentemente, outro tortricídeo, conhecido popularmente como lagarta-das-fruteiras, Argyrotaenia sphaleropa (Meyrick, 1909) (Lepidoptera: Tortricidae), também foi constatada em pomares de pessegueiro desse Estado, ocasionando entre 1,8 a 2,2\%, de frutos danificados (BOTTON et al., 2003).

Uma alternativa a ser incorporada ao manejo integrado da grafolita e da lagarta-das-fruteiras, seria a utilização do parasitóide de ovos Trichogramma pretiosum Riley, 1879 (Hymenoptera: Trichogrammatidae), que se destaca por parasitar ovos da grafolita (AFONSO, 2001; PINTO et al., 2002) e também da lagarta-das-fruteiras (BASSO et al., 1998).

\footnotetext{
'Engenheiros Agrônomos, Doutores - Departamento de Fitossanidade/DFS - Universidade Federal de Pelotas/UFPel - Campus Universitário - Cx. P. 354 96010-900 - Pelotas, RS - fgiolo.faem@hotmail.com; cristianemanzoni@hotmail.com - Bolsista do CNPq

'Engenheiro Agrônomo, Doutor, Professor Associado - Departamento de Fitossanidade/DFS - Universidade Federal de Pelotas/UFPel - Campus Universitário - Cx. P. 354 - 96010-900 - Pelotas, RS - adgrutzm@ufpel.tche.br - Bolsista de Produtividade em Pesquisa do CNPq

${ }^{3}$ Bióloga, Mestranda - Departamento de Fitossanidade/DFS - Universidade Federal de Pelotas/UFPel - Campus Universitário - Cx. P. 354 - $96010-900$ Pelotas, RS - clima@ufpel.tche.br - Bolsista de Apoio Técnico de Nível Superior do CNPq

${ }^{4}$ Aluno de Graduação em Agronomia - Universidade Federal de Pelotas/UFPel - Campus Universitário - Cx. P. 354 - $96010-900$ Pelotas, RS - wharter@ufpel.tche.br - Bolsista BIC CNPq

${ }^{5}$ Aluna de Graduação em Agronomia - Universidade Federal de Pelotas/UFPel - Campus Universitário - Cx. P. 354 - $96010-900$ Pelotas, RS - crismiler@yahoo.com.br - Bolsista PET CAPES
} 
Uma das limitações para a utilização desses parasitóides é a falta de informações sobre a seletividade dos agroquímicos, utilizados na cultura do pessegueiro para a entomofauna benéfica, que favoreceriam a integração dos métodos de controle químico e biológico. Dentre os estudos realizados, merece destaque o trabalho de Gonring et al. (1999), que avaliaram a seletividade de oito inseticidas, principalmente organofosforados, utilizados no controle da grafolita no Brasil, a vespas predadoras. Com parasitóides do gênero Trichogramma, estudo pioneiro foi realizado na Alemanha por Grützmacher et al. (2004), que avaliaram a seletividade de seis produtos fitossanitários recomendados na Produção Integrada de Pêssego (PIP) do Brasil, sobre Trichogramma cacoeciae Marchal, 1927 (Hymenoptera: Trichogrammatidae), considerada a espécie-padrão da International Organization for Biological and Integrated Control of Noxious Animals and Plants, West Palaearctic Regional Section (IOBC/ WPRS). Recentemente, Giolo et al. (2005a) avaliaram a seletividade de oito agroquímicos recomendados na PIP do Brasil, porém, para T. pretiosum.

Neste contexto, considerando o potencial e a importância de $T$. pretiosum como agente biológico de supressão populacional de G. molesta e A. sphaleropa, em pomares de pessegueiro, objetivou-se no presente estudo avaliar a seletividade de dez produtos fitossanitários utilizados nessa cultura, a adultos desse parasitóide.

\section{MATERIAL E MÉTODOS}

Os experimentos foram conduzidos conforme metodologia estabelecida pela IOBC/WPRS para Trichogramma de acordo com Hassan et al. (2000). O material biológico utilizado nos experimentos foi constituído pelo parasitóide $T$. pretiosum, coletado na região de Pelotas, RS. Esses insetos deram origem à uma criação mantida em laboratório, utilizando-se ovos inviabilizados, sob lâmpada germicida do hospedeiro Anagasta kuehniella (Zeller, 1879) (Lepidoptera: Pyralidae), à temperatura de $25 \pm 1^{\circ} \mathrm{C}$, umidade relativa do ar de $70 \pm 10 \%$ e fotofase de 14 horas.

Os estudos foram realizados com os produtos fitossanitários (g ou mL do ingrediente ativo $\left.100 \mathrm{~L}^{-1}\right)$ : 1 . abamectina $(1,44), 2$. captana (120), 3. cianamida (1560), 4 . ditianona (94), 5. etofenproxi (15), 6. glifosato $\left(6 \mathrm{~L}^{-\mathrm{ha}^{-1}}\right), 7$. mancozebe (160), 8. metoxifenozida (14,4), 9. óleo mineral (760) e 10. óxido cuproso (134), representando os tratamentos e testemunha constituída apenas por água destilada. Esses produtos estão registrados e/ou apresentaram eficácia no controle de pragas da cultura, sendo testados nas dosagens máximas registradas e/ou indicadas pelos fabricantes.

Os testes de toxicidade foram conduzidos, em laboratório, sob as mesmas condições climáticas usadas na criação do parasitóide, expondo-se adultos de $T$. pretiosum a resíduos secos dos compostos. A exposição foi feita em placas quadrangulares de vidro de $13 \mathrm{~cm}$ de lado, pulverizadas com os produtos. Depois de tratadas, as placas permaneceram à temperatura ambiente, por cerca de três horas, para secagem da calda aplicada. As aplicações foram realizadas através de pulverizadores manuais, que proporcionaram um depósito de calda de $1,75 \pm 0,25 \mathrm{mg} . \mathrm{cm}^{-2}$ da placa de vidro.

Para confinamento dos parasitóides foram confeccionadas gaiolas de exposição compostas por duas placas de vidro, contendo resíduo seco do produto, servindo como fundo e cobertura, fixas a uma moldura de alumínio composta por quatro laterais de $13 \mathrm{~cm}$ de comprimento x $1,5 \mathrm{~cm}$ de altura, através de presilhas. Em três laterais da moldura de alumínio existiam seis orifícios para ventilação ( $1 \mathrm{~cm}$ diâmetro), cobertos com tecido preto, fixado com fita adesiva para evitar a fuga dos insetos. No quarto lado haviam dois orifícios: o maior $(3,5 \mathrm{~cm} \mathrm{x} 1 \mathrm{~cm})$ foi utilizado para introdução de ovos de A. kuehniella a serem parasitados, e fornecimento de alimento para os adultos ( $3 \mathrm{~g}$ de gelatina, $100 \mathrm{~mL}$ de água e $200 \mathrm{~g}$ de mel), e o menor ( $1 \mathrm{~cm}$ diâmetro) serviu para inserção dos tubos de emergência com os indivíduos a serem testados. Essa lateral foi coberta externamente com uma fita isolante preta, protegida na altura dos referidos orifícios com outra fita adesiva evitando a adesão e imobilidade dos insetos. As superfícies externas das placas de vidro foram cobertas com papel cartão preto, possuindo um orifício central quadrangular de $10 \times 10 \mathrm{~cm}$, formando a área de contato dos insetos com o produto em teste, devido ao fototropismo dos parasitóides, tornando-os ativos na superfície de vidro exposta à luz e, conseqüentemente, em contato com a superfície contaminada.

Para a liberação dos parasitóides, no interior das gaiolas, foram utilizados tubos de emergência, sendo que cada um deles (ampola de vidro transparente de $120 \mathrm{~mm}$ de comprimento por $20 \mathrm{~mm}$ de diâmetro em uma das extremidades e $7 \mathrm{~mm}$ na outra), continha um círculo de cartolina ( $1 \mathrm{~cm}$ de diâmetro), com $250 \pm 50$ ovos de $A$. kuehniella previamente parasitados, aderido a uma tira de papel de $80 \mathrm{~mm}$ de comprimento por $15 \mathrm{~mm}$ de largura e com três finos filetes de alimento. Aproximadamente 24 horas após a emergência, os tubos contendo os adultos de T. pretiosum foram conectados às gaiolas de exposição, seis horas após a pulverização, permitindo a entrada dos 
insetos no interior da gaiola. Para acelerar a saída dos insetos dos tubos de emergência, foi acrescida a intensidade luminosa da sala e encapado o tubo de emergência, durante o período de liberação. Os tubos de emergência foram desconectados após 16 horas, e mantidos nas mesmas condições experimentais, por mais três dias, para que ocorresse a total emergência dos adultos.

O acúmulo de gases tóxicos no interior das gaiolas foi evitado empregando-se um sistema de sucção de ar constituído por duas bombas de aquário com o fluxo invertido. As bombas de aquário foram conectadas, por meio de mangueiras, às gaiolas, durante o período de condução do experimento.

Seis horas após a retirada dos tubos de emergência, cartões contendo três círculos de $1 \mathrm{~cm}$ de diâmetro contendo $450 \pm 50$ ovos inviabilizados de A. kuehniella por unidade, e alimento foram oferecidos em sobreposição às 24 (três cartões), 48 (dois cartões) e 96 (um cartão) horas após pulverização, para serem parasitados por T. pretiosum.

O número de fêmeas, em cada gaiola, foi determinado através da contagem dos ovos parasitados em cada círculo, de $1 \mathrm{~cm}$ de diâmetro, colocado no interior do tubo de emergência. $\mathrm{O}$ valor obtido foi multiplicado pelo número de parasitóides por ovo da população, e subtraído do número de adultos que permaneceram no interior do tubo de emergência, obtendo-se o número de adultos que entraram na gaiola. Esse valor foi multiplicado pela razão sexual da população, obtendo-se o número de fêmeas no interior da gaiola de exposição. Os parâmetros, número de parasitóides por ovo e razão sexual de $T$. pretiosum, foram obtidos a partir da amostragem de quatro círculos de $1 \mathrm{~cm}$ de diâmetro contendo ovos parasitados, sendo estes retirados do mesmo cartão utilizado nos tubos de emergência.

Sete dias após a aplicação dos produtos fitossanitários, as gaiolas foram desmontadas e os cartões contendo ovos do hospedeiro ofertados aos insetos em teste, retirados e acondicionados em placas de Petri de 90 x $15 \mathrm{~mm}$. Esses cartões foram mantidos nas mesmas condições ambientais por mais três dias, permitindo o desenvolvimento do parasitóide até o estágio de pupa (escurecimento característico pela deposição de grânulos de urato), para posterior contagem dos ovos parasitados. A partir do número de ovos parasitados e número de fêmeas no interior da gaiola, obteve-se o número médio de ovos parasitados por fêmea de $T$. pretiosum, para cada tratamento.

Foram utilizadas quatro repetições para cada tratamento, sendo cada gaiola de exposição considerada uma unidade experimental no delineamento inteiramente casualizado. Os dados obtidos foram testados quanto à normalidade e, submetidos à análise de variação, sendo as médias dos tratamentos comparadas com a testemunha de cada experimento pelo teste $\mathrm{F}(\mathrm{p}<0,05)$. As reduções, no número médio de ovos parasitados, em função dos produtos testados foram corrigidas pela fórmula (HASSAN et al., 2000) $R P=\left(1-R_{t} / R_{c}\right) * 100$, onde RP é a porcentagem de redução no parasitismo, $R_{t}$ é o valor do parasitismo médio para cada produto e $\mathrm{R}_{\mathrm{c}} \mathrm{o}$ parasitismo médio observado para o tratamento testemunha (negativa). Com base nas porcentagens de reduções no parasitismo, os produtos fitossanitários foram classificados segundo a IOBC/WPRS em: 1) inócuo (<30\%); 2) levemente nocivo $(30-79 \%) ; 3)$ moderadamente nocivo (80-99\%) e 4) nocivo (>99\%).

\section{RESULTADOS E DISCUSSÃO}

O número de ovos parasitados por fêmea de $T$. pretiosum, submetidas aos fungicidas captana, ditianona, mancozebe e óxido cuproso não diferiram da testemunha. Para esses fungicidas a redução no parasitismo foi, no máximo de 21,69\%, sendo enquadrados na categoria inócuo (classe 1) de acordo com a IOBC/WPRS (Tabela 1). Resultados similares foram relatados por Giolo et al. (2005a) para captana na mesma concentração testada no presente trabalho, com T. pretiosum. Por outro lado, Hassan et al. (1998) obtiveram 49,10\% de redução no parasitismo por $T$. cacoeciae, com o uso de captana $83 \mathrm{~W}$, que foi enquadrado na classe 2 (levemente nocivo). As diferenças obtidas neste caso, provavelmente devem-se à concentração de ingrediente ativo de 5,68 vezes superior à que foi testada no presente estudo. Hassan (1998) obteve que ditianona a 0,2\% foi inócuo para T. cacoeciae. Giolo et al. (2005a), testando mancozebe na mesma concentração avaliada nesse trabalho sobre $T$. pretiosum, obtiveram uma redução de $13 \%$ no número de ovos parasitados, classificando o produto como inócuo. Por outro lado, Hassan (1998), testando mancozebe para $T$. cacoeciae, na mesma concentração testada neste trabalho, obteve a classe moderadamente nociva (classe 3). Diferenças essas, provavelmente relacionadas às características intrínsecas de cada formulação comercial.

Para o acaricida-inseticida abamectina, o número de ovos parasitados foi significativamente inferior, ocasionando redução de $100 \%$, no parasitismo por $T$. pretiosum (Tabela 1). Resultado distinto foi observado por Rocha \& Carvalho (2004), em estudo visando à adaptação da metodologia da IOBC/WPRS para condições brasileiras, com $T$. pretiosum. Observou-se que, na concentração de $0,0018 \%$ de ingrediente ativo, portanto superior à testada 
no presente estudo $(0,00144 \%), 81,40 \%$ de redução no parasitismo, classificaram abamectina como moderadamente nociva (classe 3), sem fazer menção da formulação comercial avaliada. Assim, as diferenças encontradas poderão estar relacionadas à formulação, variações na metodologia empregada ou à diferenças de suscetibilidade, entre as linhagens do parasitóide avaliadas.

O inseticida metoxifenozida foi inócuo ao parasitóide (classe 1), não ocasionando redução no parasitismo por $T$. pretiosum (Tabela 1). Além desse aspecto, esse inseticida é relatado por Arioli et al. (2004) como um produto eficiente no controle da grafolita, podendo ser uma alternativa empregada em programas de manejo integrado de insetos-praga, na cultura do pessegueiro. Classificação similar foi obtida por Grützmacher et al. (2004), testando outra formulação comercial de metoxifenozida sobre $T$. cacoeciae. Resultado distinto foi obtido por Rocha \& Carvalho (2004), que testaram metoxifenozida sobre adultos de $T$. pretiosum, na concentração de $0,012 \%$ do ingrediente ativo, portanto, inferior à utilizada neste trabalho $(0,0144 \%)$ e constataram uma redução de $89,10 \%$ no parasitismo, classificando-o como moderadamente nocivo (classe 3). Essa divergência de resultados, provavelmente está relacionada à variações na metodologia aplicada em cada estudo. Além disso, diferenças de suscetibilidade intraespecífica de produtos fitossanitários à T. pretiosum, já foram relatadas na literatura (CARVALHO et al., 2003) e também podem ter contribuído para as discrepâncias obtidas entre ambos os estudos.

O óleo mineral avaliado reduziu em 70,76\% o parasitismo, sendo, portanto, levemente nocivo (classe 2). Testando outro óleo mineral também recomendado para a cultura do pessegueiro, Giolo et al. (2005a) obtiveram $89,51 \%$ de redução no número de ovos parasitados por $T$. pretiosum, classificando esse produto como moderadamente nocivo (classe 3). As diferenças obtidas entre os dois óleos minerais, provavelmente devem-se à dosagem recomendada, apesar dos dois produtos possuírem concentrações de ingrediente ativo semelhantes.

TABELA 1 - Número médio ( \pm EP) de ovos de Anagasta kuehniella, parasitados por Trichogramma pretiosum, redução (\%) no parasitismo em função da exposição a produtos fitossanitários e classificação da seletividade desses produtos. Temperatura $25 \pm 1^{\circ} \mathrm{C}$; UR: $70 \pm 10 \%$; fotofase: 14 horas.

\begin{tabular}{|c|c|c|c|c|c|}
\hline \multirow[b]{2}{*}{ Produto fitossanitário } & \multirow[b]{2}{*}{$\mathbf{D C}^{1}$} & \multicolumn{2}{|c|}{$\mathrm{N}^{\circ}$ ovos parasitados fêmea } & \multirow[b]{2}{*}{$\mathbf{R} \mathbf{P}^{3}$} & \multirow[b]{2}{*}{ Classes $^{4}$} \\
\hline & & $\begin{array}{c}\text { Produto } \\
\text { fitossanitário }^{2}\end{array}$ & Testemunha & & \\
\hline Abamectina & 1,44 & $0,00 \pm 0,00^{*}$ & $32,61 \pm 2,02$ & 100,00 & 4 \\
\hline Captana & 120 & $25,15 \pm 2,55^{\text {n.s. }}$ & $27,51 \pm 3,39$ & 8,58 & 1 \\
\hline Cianamida & 1560 & $0,25 \pm 0,20^{*}$ & $28,16 \pm 3,77$ & 99,11 & 4 \\
\hline Ditianona & 94 & $29,00 \pm 2,07^{\text {n.s. }}$ & $26,83 \pm 0,31$ & 0,00 & 1 \\
\hline Etofenproxi & 15 & $0,00 \pm 0,00^{*}$ & $32,61 \pm 2,02$ & 100,00 & 4 \\
\hline Glifosato & 6 L.ha $^{-1}$ & $9,67 \pm 4,08^{*}$ & $27,51 \pm 3,39$ & 64,85 & 2 \\
\hline Mancozebe & 160 & $25,81 \pm 3,97^{\text {n.s. }}$ & $32,96 \pm 6,06$ & 21,69 & 1 \\
\hline Metoxifenozida & 14,4 & $43,58 \pm 3,15^{*}$ & $32,61 \pm 2,02$ & 0,00 & 1 \\
\hline Óleo mineral & 760 & $11,75 \pm 5,17^{*}$ & $40,19 \pm 2,27$ & 70,76 & 2 \\
\hline Óxido cuproso & 134 & $22,61 \pm 6,03^{\text {n.s. }}$ & $27,51 \pm 3,39$ & 17,81 & 1 \\
\hline
\end{tabular}

${ }^{1} \mathrm{DC}=$ Dosagem $\left(\mathrm{g}\right.$ ou $\mathrm{mL}$ da formulação comercial.100 L $\left.\mathrm{L}^{-1}\right) .{ }^{2 *}=$ significativamente diferentes pelo teste $\mathrm{F}(\mathrm{p}<0,05),{ }^{\mathrm{n} . \mathrm{s}}=$ não significativo. ${ }^{3} \mathrm{RP}=$ Redução no parasitismo comparado com a testemunha. ${ }^{4}$ Classes da IOBC/WPRS para teste de seletividade com adultos de Trichogramma: $1=$ inócuo $(<30 \%), 2=$ levemente nocivo $(30-79 \%), 3=$ moderadamente nocivo $(80-99 \%)$, 4=nocivo $(>99 \%)$. 
O inseticida etofenproxi, do grupo químico dos éteres difenílicos, foi classificado como nocivo (classe 4), causando redução de $100 \%$ no parasitismo, embora encontrando-se em fase de registro para controle da grafolita, na cultura do pessegueiro (ARIOLI et al., 2004).

O herbicida glifosato reduziu em $64,85 \%$ o número de ovos parasitados, sendo classificado como levemente nocivo (classe 2). Giolo et al. (2005b), testando oito diferentes formulações comerciais de glifosato sobre $T$. pretiosum, obtiveram para este mesmo composto, redução de $86,42 \%$ no parasitismo, classificando-o como moderadamente nocivo (classe 3 ). A diferença obtida, provavelmente, se deve à concentração de equivalente ácido de glifosato testada, sendo 33,33\% superior à avaliada no presente estudo $(1,08 \%)$. O regulador de crescimento de plantas cianamida reduziu em $99,11 \%$, o número de ovos parasitados por $T$. pretiosum, sendo considerado nocivo (classe 4).

De acordo com os resultados obtidos nesse estudo e, seguindo a metodologia de testes da IOBC/WPRS (HASSAN \& ABDELGADER, 2001) para T. cacoeciae, os fungicidas captana, óxido cuproso, ditianona e mancozebe e o inseticida metoxifenozida foram seletivos ao parasitóide T. pretiosum, não sendo necessário testes nas etapas subseqüentes nos estágios imaturos, persistência biológica e condições de campo. Para os compostos abamectina, cianamida, etofenproxi, glifosato e óleo mineral, os resultados obtidos em laboratório não devem ser extrapolados para condições de campo e devem prosseguir na seqüência de testes, como sugerido por Hassan \& Abdelgader (2001).

\section{CONCLUSÕES}

A partir dos resultados obtidos nos testes de toxicidade, com as respectivas dosagens indicadas dos produtos fitossanitários para a cultura do pessegueiro, conclui-se que os fungicidas captana, óxido cuproso, ditianona e mancozebe e o inseticida metoxifenozida foram inócuos. $\mathrm{O}$ inseticida à base de óleo mineral e o herbicida glifosato foram levemente nocivos. O acaricida-inseticida abamectina, o inseticida etofenproxi e o regulador de crescimento de plantas cianamida foram nocivos à $T$. pretiosum.

\section{AGRADECIMENTOS}

Ao Conselho Nacional de Desenvolvimento Científico e Tecnológico (CNPq) e à Fundação de Amparo à Pesquisa do Estado do Rio Grande do Sul (FAPERGS), pelo apoio financeiro para a realização da pesquisa.

\section{REFERÊNCIAS BIBLIOGRÁFICAS}

AFONSO, A. P. S. Controle da Grapholita molesta (Busck, 1916) (Lepidoptera: Tortricidae) no sistema de produção integrada de pêssego. 2001. 62 f. Dissertação (Mestrado em Fitossanidade - Entomologia) - Universidade Federal de Pelotas, Pelotas, 2001.

ARIOLI, C. J.; BOTTON, M.; CARVALHO, G. A. Controle químico da Grapholita molesta (Busck) (Lepidoptera: Tortricidae) na cultura do pessegueiro. Ciência Rural, Santa Maria, v. 34, n. 6, p. 1695-1700, 2004.

BASSO, C.; GRILLE, G.; POMPANON, F.; ALLEMAND, R.; PINTUREAU, B. Comparación de los caracteres biológicos y etológicos de Trichogramma pretiosum y de T. exiguum (Hymenoptera: Trichogrammatidae). Revista Chilena de Entomologia, Santiago, v. 25, n. 1, p. 45-53, 1998.

BOTTON, M.; ARIOLi, C.; COllettA, V. D. Monitoramento da mariposa oriental Grapholita molesta (Busck, 1916) (Lepidoptera: Tortricidae) na cultura do pessegueiro. Bento Gonçalves: Embrapa CNPUV, 2001. 4 p. (Comunicado técnico, 38).

BOTTON, M.; BAVARESCO, A.; GARCIA, M. S. Ocorrência de Argyrotaenia sphaleropa (Meyrick) (Lepidoptera: Tortricidae) danificando pêssegos na Serra Gaúcha, Rio Grande do Sul. Neotropical Entomology, Londrina, v. 32, n. 3, p. 503-505, 2003.

CARVALHO, G. A.; PARRA, J. R. P.; BAPTISTA, G. C. Bioatividade de produtos fitossanitários utilizados na cultura do tomateiro (Lycopersicum esculentum Mill.) a Trichogramma pretiosum Riley, 1879 (Hymenoptera: Trichogrammatidae) nas gerações $F_{1}$ e $F_{2}$. Ciência e Agrotecnologia, Lavras, v. 27, p. 261-270, 2003.

GIOLO, F. P.; GRÜTZMACHER, A. D.; MANZONI, C. G.; NÖRNBERG, S. D.; STEFANELLO JÚNIOR, G. J. Seletividade de agrotóxicos indicados na produção integrada de pêssego a Trichogramma pretiosum Riley, 1879 (Hymenoptera: Trichogrammatidae). Revista Brasileira de Fruticultura, Jaboticabal, v. 27, n. 2, p. 222-225, 2005 a.

GIOLO, F. P.; GRÜTZMACHER, A. D.; PROCÓPIO, S. O.; MANZONI, C. G.; LIMA, C. A. B.; NÖRNBERG, S. D. Seletividade de formulações de glyphosate a Trichogramma pretiosum (Hymenoptera: Trichogrammatidae). Planta Daninha, Viçosa, v. 23, n. 3, p. 457-462, 2005b. 
GONRING, A. H. R.; PICANÇO, M.; MOURA, M. F.; BACCI, L.; BRUCKNER, C. H. Seletividade de inseticidas, utilizados no controle de Grapholita molesta (Busck) (Lepidoptera: Olethreutidae) em pêssego, a vespidae predadores. Anais da Sociedade Entomológica do Brasil, Londrina, v. 28, n. 2, p. 301-306, 1999.

GRÜTZMACHER, A. D.; ZIMMERMANN, O.; YOUSEF, A.; HASSAN, S. A. The side-effects of pesticides used in integrated production of peaches in Brazil on the egg parasitoid Trichogramma cacoeciae Marchal (Hym.; Trichogrammatidae). Journal of Applied Entomology, Berlin, v. 128 , n. 6 , p. 377-383, 2004.

HASSAN, S. A. The side-effects of 161 pesticides on the egg parasitoid Trichogramma cacoeciae Marchal (Hymenoptera: Trichogrammatidae). Egg Parasitoids, Cali, p. 63-76, 1998

HASSAN, S. A.; ABDELGADER, H. A sequential testing program to assess the effects of pesticides on Trichogramma cacoeciae Marchal (Hym.; Trichogrammatidae). Bulletin IOBC/WPRS, Darmstadt, v. 24 , p. $71-81,2001$.

HASSAN, S. A.; HAFES, B.; DEGRANDE, P. E.; HERAI, $\mathrm{K}$. The side-effects of pesticides on the egg parasitoid Trichogramma cacoeciae Marchal (Hym.; Trichogrammatidae), acute dose-response and persistence tests. Journal of Applied Entomology, Berlin, v. 122, n. 9, p. 569-573, 1998.
HASSAN, S. A.; HALSALL, N.; GRAY, A. P.; KUEHNER, C.; MOLL, M.; BAKKER, F. M.; ROEMBKE, J.; YOUSEF, A.; NASR, F.; ABDELGADER, H. A laboratory method to evaluate the side effects of plant protection products on Trichogramma cacoeciae Marchal (Hym.; Trichogrammatidae). In: CANDOLFI, M. P.; BLÜMEL, S.; FORSTER, R.; BAKKER, F. M.; GRIMM, C.; HASSAN, S. A.; HEIMBACH, U.; MEAD-BRIGGS, M. A.; REBER, B.; SCHMUCK, R.; VOGT, H. (Eds.). Guidelines to evaluate side-effects of plant protection products to non-target arthropods. Gent: IOBC/WPRS, 2000. p. 107-119.

INSTITUTO BRASILEIRO DE GEOGRAFIA E ESTATÍSTICA. Produção agrícola municipal: culturas temporárias e permanentes. Disponível em: <http:// www.sidra.ibge.gov.br/bda>. Acesso em: 23 jun. 2005.

PINTO, J. D.; KOOPMANSCHAP, A. B.; PLATNER, G. R.; STOUTHAMER, R. The North American Trichogramma (Hymenoptera: Trichogrammatidae) parasitizing certain Tortricidae (Lepidoptera) on apple and pear, with ITS2 DNA characterizations and description of a new species. Biological Control, San Diego, v. 23, n. 2, p. 134-142, 2002.

ROCHA, L. C. D.; CARVALHO, G. A. Adaptação da metodologia padrão da IOBC para estudos de seletividade com Trichogramma pretiosum Riley, 1879 (Hymenoptera: Trichogrammatidae) em condições de laboratório. Acta Scientarium, Maringá, v. 26, n. 3, p. 315-320, 2004. 